\title{
CONHECIMENTO DAS MULHERES E DOS HOMENS REFERENTEAO CLIMATÉRIO E MENOPAUSA
}

Laiany Lais de Alcântara ${ }^{1}$

Leila Cristine do Nascimento ${ }^{1}$

Vânia Aparecida da Costa Oliveira ${ }^{1}$
ORCID: https://orcid.org/0000-0002-6557-2910

ORCID: https://orcid.org/0000-0003-4744-4576

ORCID: https://orcid.org/0000-0001-7082-5997

Objetivo: conhecer o que as mulheres e os homens com vínculo trabalhista ou estudantil da Universidade Federal de São João del-Rei, Campus Centro-Oeste, sabem sobre o climatério e menopausa. Método: estudo de caso único, com abordagem qualitativa e fundamentada na técnica de Análise Crítica do Discurso. A pesquisa contou com 43 participantes, sendo 15 mulheres e 28 homens. Resultados: a distinção entre climatério e menopausa nem sempre é clara para os participantes, sendo desconhecidas, especialmente, pelos participantes do sexo masculino. Além disso, o climatério é um periodo em que as mulheres queixam-se de vários sintomas, tanto biológicos quanto psicológicos. Considerações finais: há uma defasagem no conhecimento das mulheres referente ao climatério e à menopausa. Nota-se o desconhecimento total dos homens referente ao climatério, e poucos sabem o que é a menopausa. Em face ao exposto, evidencia-se a necessidade de mais estudos que busquem explorar a temática menopausa/climatério.

Descritores: mulher; homem; conhecimento; climatério; menopausa.

\section{WOMEN AND MEN'S KNOWLEDGE REGARDING THE CLIMATE AND MENOPAUSE}

Objective: to know the that women and men with a labor or student bond of the Federal University of São João del-Rei, Campus Central-West, know about the climacteric and menopause. Method: a unique case study, with a qualitative approach and based on the technique of Critical Discourse Analysis. The survey counted on 43 participants, being 15 women and 28 men. Results: the distinction between the phases of female life understood by the climacteric and the menopause is not always clear to the participants, being unknown, especially, by the male participants. In addition, it is a time when women complain of various symptoms, both biological and psychological. Conclusion: there is a lag in women's knowledge regarding such periods. Men's total ignorance of the climacteric is noted, and few know what menopause is. In view of the above, it is evident the need for further studies that seek to explore the menopausal / climacteric theme.

Descriptors: woman; man; knowledge; climacteric; menopause.

\section{CONOCIMIENTO DE LAS MUJERES Y DE LOS HOMBRES REFERENTEAL CLIMATERAL Y MENOPAUSA}

Objetivo: conocer el desarrollo que las mujeres y los hombres con vinculo laboral o estudiantil de la Universidad Federal de San Juan del Rey, Campus Centro-Oeste, saben sobre el climaterio y la menopausia. Metodo: estudio de caso único, con abordaje cualitativo y fundamentado en la técnica de Análisis Crítico del Discurso. La encuesta contó con 43 participantes, siendo 15 mujeres y 28 hombres. Resultados: la distinción entre las fases de la vida femenina comprendidas por el climaterio y la menopausia no siempre es clara para los participantes, siendo desconocidas, especialmente, por los participantes del sexo masculino. Además, es un periodo en que las mujeres se quejan de varios síntomas, tanto biológicos y psicológicos. Conclusión: hay un desfase en el conocimiento de las mujeres referente a tales periodos. Se nota el desconocimiento total de los hombres referente al climaterio y pocos saben lo que es la menopausia. En vista de lo expuesto se evidencia la necesidad de más estudios que busquen explorar la temática menopausia / climaterio

Descriptores: mujer; hombre; conocimiento; climaterio; menopausia.

IUniversidade Federal de São João Del-Rei, MG.

Autor Correspondente: Laiany Alcântara E-mail: laiany.alcantara@hotmail.com

Recebido: 24/06/2019

Aceito: 14/08/2019 


\section{INTRODUÇÃO}

A população feminina representa $51,6 \%$ da população brasileira $^{(1)}$, tal fato reforça a necessidade de políticas públicas que contemplem as especificidades inerentes à saúde feminina e que tenham como foco a integralidade da assistência. Dessa forma, a Política Nacional de Assistência Integral à Mulher (PNAIM) foi instituida pelo Ministério da Saúde, com o objetivo de ampliar o foco da assistência à saúde da mulher que, até então, era limitada às demandas relativas à gravidez e ao parto e, assim, ratificar uma visão restrita da mulher(2).

Considerando-se a ampliação da assistência à saúde da mulher e as diversas especificidades dessa população, sobretudo, o impacto que o aumento da expectativa de vida tem sobre a sua saúde, o climatério e a menopausa despontam como fases importantes e que necessitam de maiores cuidados, tendo em vista a melhoria da qualidade de vida durante e após essas fases.

O climatério corresponde à transição entre o período reprodutivo e o não reprodutivo da mulher, a faixa etária de acontecimento compreende mulheres de 35 a 65 anos. Já a menopausa é reconhecida após 12 meses consecutivos de amenorreia, assim é considerada um evento dentro do climatério. Este, passa, então, a ser delimitado por duas fases assim definidas: pré-menopausa ou perimenopausa e a pósmenopausa. ${ }^{(4-3)}$

$\mathrm{Na}$ fase de pré-menopausa sintomas comuns são: a irregularidade dos ciclos menstruais, menorragia ou hipermenorreia. Os fogachos e alterações de humor também podem iniciar. Na fase pós-menopausa algumas mudanças podem ser notadas como o ressecamento vaginal, dispareunia, urgência urinária, disúria, perda da libido, dentre outras. É de grande relevância citar também os aspectos psicológicos dessas mulheres, pois, o fato de estar no climatério, especialmente na pós-menopausa, pode representar um período de perda da feminilidade, produtividade e de grande desconforto. Esses sintomas variam de mulher para mulher e serão vivenciados de acordo com a história de vida de cada uma, levando em conta as condições sociais, econômicas, fatores hereditários e culturais. ${ }^{(4-3)}$

Nesse contexto, diante das modificações endócrinas, fisicas e emocionais que ocorrem durante essas fases, fazse necessário descobrir o que as mulheres sabem a respeito do assunto e outras questões relacionadas. Além disso, é de extrema importância identificar quais os conhecimentos que os homens possuem a respeito desses períodos, pois, seja no papel de companheiro, seja no papel de filhos ou demais familiares, o não conhecimento desse período por parte dos homens pode levar ao julgamento e repressão da mulher que passa por essa fase, causando ou piorando um quadro de um sofrimento emocional.
Diante disso, a criação de estratégias para melhorar o conhecimento de homens e mulheres acerca do climatério e da menopausa pode contribuir para que essas fases sejam vivenciadas com mais segurança, apoio, companheirismo e solidariedade. Considerando-se a complexidade inerente a essas, torna-se necessário que elas façam parte do rol de capacitações dos profissionais de saúde e dos currículos dos cursos dessa área, pois, só assim, pode-se garantir uma assistência mais humanizada e integral às mulheres e, consequentemente, uma melhoria na qualidade de vida.

Assim sendo, este estudo tem como objetivo conhecer - que as mulheres e os homens com vínculo trabalhista ou estudantil da Universidade Federal de São João delRei, Campus Centro-Oeste (UFSJ/CCO), sabem sobre o climatério e menopausa.

\section{METODOLOGIA}

\section{Tipo de estudo}

Estudo de caso único, com abordagem qualitativa e utilização da técnica de Análise Crítica do Discurso (ACD), proposta pelo linguista britânico Norman Fairclough. A ACD propõe analisar o papel da linguagem como reprodução de prática social e ideologias, além do seu papel essencial na transformação social. Para Fairclough, por um lado o discurso é moldado pela estrutura social e, por outro lado, constitutivo de uma estrutura socia( ${ }^{(5)}$. Por sua vez, o estudo de caso único permite realizar entrevistas com as pessoas envolvidas num determinado evento, permitindo assim, investigar em profundidade e no contexto de vida real(b).

\section{Participantes da pesquisa}

A pesquisa contou com 43 participantes, sendo 15 mulheres e 28 homens. Obedeceu-se aos critérios de inclusão: a) ter vínculo estudantil ou trabalhista com a UFSJ/ CCO; b) ter idade entre 35 e 65 anos (mulheres); ter mais de 18 anos de idade (homens).

\section{Local do estudo}

A pesquisa foi realizada no Campus da UFSJ/CCO, Dona Lindu.

\section{Coleta de dados}

Os dados foram coletados por meio de roteiro semiestruturado, composto por onze perguntas abertas para as mulheres e cinco para os homens. E em ambos, apresentavam perguntas fechadas com o objetivo de conhecer o perfil sociodemográfico.

Antes da aplicação do questionário foi realizado um teste piloto, com oito pessoas, estes não foram considerados nos resultados. As entrevistas ocorreram de agosto a novembro de 2018, o tempo médio das entrevistas foi de quatro minutos. 
A captação dos participantes foi por meio de busca ativa e agendamento por meio de endereço eletrônico. A aplicação do questionário foi feita após a explicação do projeto de pesquisa, leitura e assentimento do participante formalizado no Termo de Consentimento Livre e Esclarecido (TCLE). O local utilizado foram salas de aula desocupadas, garantindo a privacidade e conforto aos participantes.

Não houve um número determinado de respondentes. A coleta de dados se encerrou quando ocorreu saturação dos dados que, neste estudo, foi por replicação literal(6). A saturação foi percebida na entrevista 37. Foram realizadas mais seis entrevistas que confirmaram a saturação, encerrando a coleta de dados na entrevista 43. Estas foram identificadas por código numérico, garantindo o anonimato dos participantes. Dessa forma, foi padronizada a utilização da primeira letra corresponde ao curso do entrevistado ou vínculo trabalhista. Exemplo: B (bioquímica); T (trabalhador); P (professor); seguido pelas letras F (feminino) ou M (masculino), e por último o número correspondente à entrevista, como por exemplo: BFOl (bioquímica, feminino, entrevista 01).

\section{Procedimento de análise dos dados}

As entrevistas foram transcritas na integra. Para Fairclough(5), a transcrição determina, necessariamente, a interpretação da fala, desse modo, a transcrição é vista como uma teoria. Após isso, foram lidas e analisadas de forma compreensiva, buscando identificar discursos semanticamente semelhantes, que possam ser relacionados entre si, dando origem às categorias.

\section{Procedimentos éticos}

Seguiu-se as recomendações da Resolução 466/2012 do Conselho Nacional de Saúde, referentes às pesquisas que envolvem seres humanos, foi aprovado pelo Comitê de Ética em Pesquisa (CEP) da UFSJ/CCO, por meio do parecer consubstanciado número: 2.616.035, CAAE: 3250418.9.0000.55458.

\section{RESULTADOS}

A análise dos dados acerca do conhecimento de mulheres e homens em relação ao climatério e menopausa possibilitou a construção de cinco categorias empíricas:

\section{Climatério e Menopausa: Dois periodos distintos reconhecidos como o mesmo \\ É central nos discursos dos participantes que nem sempre é clara a distinção entre o climatério e a menopausa, sendo ressaltado a cessação da menstruação como o marco de tais períodos, como pode-se observar nos discursos abaixo:}

"O término do ciclo menstrual da mulher" FM006

“É a menopausa. É um momento em que a mulher passa por várias alterações.[..] É a cessação da menstruação [...]" PF040

"Uai, eu acho que é o mesmo, menopausa e climatério [...] Essa mudança também, eu não vejo muita diferença na colocação das duas não"BFOO2

A expressão "Eu acho", presente no discurso de BF002, revela uma incerteza do que significa e o que marca as fases. Essa incerteza é ratificada na expressão "eu não vejo muita diferença". Evidencia-se assim que, para BFOO2, o climatério e a menopausa são sinônimos.

\section{Climatério e menopausa: uma fase que transcende os aspectos biológicos}

Nesta categoria, os participantes expressam sintomas de frustração causados pelo fim da fase reprodutiva, há uma sensação de perda da essência feminina. Os discursos abaixo ilustram essa assertiva:

"Elas se sentem impotentes por não poder mais ter filhos, acham que a fase sexual diminuem, elas se sentem com a auto estima baixa, por que a mulher não vai menstruar mais então ela vai se sentir diminuida perante as outras mulheres" EMOII

"[..] é uma fase de conflito mesmo, porque como você está deixando de reproduzir, você perde a capacidade de gestação de engravidar [...]" PF037

"[...] Eu queria ter outro filho ai a gente fica meio triste desse período chegar e eu não ter tido[...]" DEO4O

Nota-se também nos discursos das participantes o impacto do climatério e menopausa na vida conjugal:

"Vejo muito elas reclamando da questão de intimidade com o marido[...], que muita das vezes o marido não tem essa noção que para ela a coisa não está confortável[...] "BMO24

"[..]Muitas relatam que perdem o desejo de ter relação sexual e muitas ficam triste por isso. Porque pode ocorrer alguma infidelidade no casamento e elas se sentem culpadas por isso [...] E elas ficam depressivas por causa disso" EMO4O

A crença da perda da feminilidade e a associação do climatério e da menopausa ao envelhecimento é presente nas falas: 
"Todas as mulheres que eu conheço que entram nessafase, elas têm um pouco de frustração, é como se elas tivessem perdendo um pouco "do que é ser mulher". Porque existe uma relação entre a ovulação a menstruação e "o que é ser mulher", elas pensam “ agora eu estou envelhecendo", existe ainda no nosso país a relação que a terceira idade nãotemumaboa relaçãocom sexualidade, comsermulher, com beleza. Então eu não vejo as mulheres enfrentando o climatério positivo, fala-se muito dos transtornos físicos que ele traz, mas acho que além disso o significado desse final de ciclo reprodutivo e talvez esteja relacionado sim com o desejo, envelhecimento"PFO43

"[...]É como se fosse uma sensação de envelhecimento. para todas. Então eu acho que ela precisa estar preparada $e$ isso precisa ser tranquilo porque é fisiológico, mas eu acho que uma grande parte delas tem essa sensação de envelhecimento, [...] deixar de ter essa característica feminina que é reprodução"PF037

É frequente nas falas das participantes a expressão "todas". O uso desse termo evidencia que, de forma geral, as mulheres se sentem frustradas ao entrar no periodo do climatério ou da menopausa, como relatado na fala da PF043. Já na fala da PF037, o uso da palavra "todas" vem relacionado ao que ela acha que acontece quando as mulheres entram no climatério ou na menopausa, e ela generaliza afirmando que todas as mulheres vão vivenciar uma sensação de envelhecimento.

\section{Climatério e Menopausa: Principais sinais e sintomas vivenciados pelas mulheres}

Percebe-se que os principais sintomas relatados foram: fogacho, a labilidade de humor, perda da libido, dor em membros inferiores, irregularidade do ciclo menstrual e enxaqueca:

"Só mesmo a variação de humor [...]" PF042

"No início porvolta dos 52 anos eu tive essa instabilidade do humor mais acentuada, o ganho de peso mais abdominal, o ressecamento vaginal com a dispareunia e a enxaqueca. $E$ as ondas de calor foram bem exacerbadas no início e com o passar do tempo elas foram diminuindo[...]"PFO41

"[...] foi tranquilo, eu tive essas ondas de calor, tive também uma diminuição da libido[..]"PFO32

Pode-se notar que PF04l usa no seu discurso o advérbio de intensidade "bem", que permite inferir o quão intenso são esses sintomas. Já PF032, revela no seu discurso que tem vivenciado esse momento de forma "tranquila". Portanto, os diferentes discursos mostram que há uma singularidade na forma como cada mulher vivencia e sente esses sintomas. Essa assertiva é reiterada pelo discurso de TF004:

“Eu posso te falar de experiência própria, né? Então como sente: por um lado muito bem, por que a gente tá nessa idade, mas quando dá mudança hormonal assim, muita coisa muda [...]"TFOO4

\section{Climatério: Um mundo desconhecido pelos homens}

Os discursos a seguir evidenciam que a maioria dos homens não sabem o que é climatério:

“Desculpe, mas eu nunca ouvi o termo"BMO19 “Não faço a menor ideia" BM034

“Não sei “EMO27

O uso dos advérbios de negação "não" e "nunca" descreve a realidade sobre o desconhecimento dos homens sobre o climatério, e esse desconhecimento os impede de auxiliar as mulheres que vivenciam essa fase, como mostram os discursos abaixo:

“Ah, muita pouca coisa porque é um ciclo natural, né?

Então a gente não sabe o que fazer"FM006

"Não sei. Eu não sei o que fazer" TM033

\section{Climatério: 0 que as mulheres fazem para melhorar a qualidade de vida?}

A partir dos discursos fica evidente que as mulheres desconhecem medidas alternativas que podem minimizar os sintomas e melhorar a qualidade de vida:

\section{“Eu lavava o rosto na água fria"TFEO43}

"Me ensinaram que levantar os braços para cima fala que o sangue corre e que passa. Ai eu faço é isso[...]"TF004

Outros discursos permitem inferir que o profissional médico, sobretudo o ginecologista, é ainda o mais requisitado pelas mulheres para esclarecer dúvidas a respeito do climatério e da menopausa.

“Ginecologista, somente ele" TFOO4 “

[...]Tem a minha ginecologista" PFEO42

\section{DISCUSSÃO}

Como apresentado nos resultados, menopausa e climatério são, comumente, confundidos ou simplesmente identificados como um mesmo período. E, embora sejam fases que acometem todo o público feminino em uma determinada 
etapa de sua vida, tais fases não podem ser apresentadas de forma padronizada, pois, o mesmo período pode ter diversos significados e alterações diferentes em cada mulher ${ }^{(4)}$.

A ausência de conhecimento sobre esses períodos impacta diretamente no autocuidado da mulher. Um estudo,(7) realizado no ano de 2015, com mulheres que se encontravam na pós menopausa, mostrou que apenas 33,3\% delas haviam sido preparadas para a vivência desta etapa da vida, a maioria por orientação médica. $26.6 \%$ afirmaram que gostariam de terem sido preparadas com antecedência, pois consideram que não se cuidaram como deveriam. Esses dados corroboram com a pesquisa, uma vez que a maior parte das entrevistadas afirmaram não procurar nenhum tipo de ajuda profissional, a fim de esclarecer dúvidas ou buscar informações sobre climatério e menopausa .

Dessa forma, o climatério para muitas mulheres é um período que possui uma imagem negativa preponderante em relação às demais fases da vida. Pois, "representa a chegada da etapa mais experiente da mulher, com a perda dos caracteres da juventude, diminuição da fecundidade e o aparecimento de sintomas que podem comprometer a autoestima e interferir na qualidade de vida" (8:69)

Além dos aspectos físicos, o climatério e a menopausa também impactam na vida social da mulher. Um estudo,(9) realizado em 2017, mostrou que $81,2 \%$ das mulheres referiram algum sintoma vasomotor, sendo o mais prevalente 0 fogacho. Quando se trata de sintomas urogenitais, 56,2\% das mulheres referiram a diminuição da libido, o que pode afetar profundamente a interação com seus companheiros. No que diz respeito aos sintomas psicológicos, 31 (96,9\%) mulheres apresentaram pelo menos um dos sintomas, sendo os mais prevalentes a diminuição da memória e concentração, seguidos da ansiedade e fadiga.

Outra pesquisa, realizada com 50 mulheres na faixa etária entre 40 a 65 anos apontou que mulheres com idade entre 56 a 65 anos, (40\%) dizem ter nenhuma ou muito pouca informação sobre a menopausa e tratamentos. Dentre todas as participantes, 99\% manifestaram três ou mais sintomas do climatério/menopausa, $50 \%$ sentem depressão, $66,6 \%$ fogachos, $23,3 \%$ dores musculares ou articulares, $20 \%$ diminuição da libido e 13,3\% tiveram alterações físicas. Ademais, apontou que as mulheres entrevistadas se mostraram desejosas em conhecer melhor sobre essa fase(10).

Assim sendo, durante o climatério e a menopausa, sintomas psicológicos podem causar um impacto significativo na qualidade de vida. Como a ansiedade, que está ligada a sentimentos de medo, de tensão, e pode ser causada por contato com algo novo, em situações inusitadas ou desconhecidas, como é o caso do climatério, que para muitas mulheres é uma fase desconhecida. Além disso, tem-se encontrado relação entre sintomas psicológicos e as doenças, isto é, as emoções podem contribuir para o aparecimento de doenças específicas ou também podem ser consequência de doenças, desse modo podem acabar influenciando no seu prognóstico(11).

Outro ponto que merece destaque é o desconhecimento dos homens referente ao climatério e menopausa. Tal fato é demonstrado em um estudo ${ }^{(12)}$ de 2013, com homens. Neste estudo, pode-se identificar que eles possuem dificuldades para falar sobre o tema e que quando questionados são bem pontuais nas respostas. Outro aspecto importante é que eles reconhecem que a esposa necessita de apoio, mas que não sabem o que fazer para ajudá-las. Ressalta-se que o suporte familiar, sobretudo, do companheiro, é fundamental, pois, este, normalmente está mais próximo da mulher.

Diante do exposto, é essencial que os profissionais de saúde compreendam a subjetividade de cada mulher, reconheçam suas necessidades que algumas vezes não são verbalizadas, buscando o companheiro ou familiar, para que participe das consultas e também das mudanças de hábitos quando necessário. Desse modo,a equipe de saúde assegura que o diagnóstico e o acompanhamento sejam adequados(3).

\section{Limitações do estudo}

Ressalta-se a dificuldade de encontrar estudantes acima de 35 anos, visto que o público da Universidade em geral é jovem.

\section{Contribuição do estudo para a prática}

Os resultados encontrados servem como subsídios para o planejamento de novas ações para esse público que poderão contribuir para melhoria do cuidado.

\section{CONSIDERAÇÕES FINAIS}

Os resultados do estudo revelam que a maior parte das mulheres não sabem ao certo do que se trata o climatério e a menopausa. Ademais, predominantemente, não buscam ajuda de profissionais de saúde, e quando buscam, procuram, apenas, o ginecologista. Além disso, não oportunizam o momento da consulta para falarem de forma mais profunda sobre esses períodos. Pode-se verificar, também, que grande parte das mulheres não realizam atividades alternativas como forma de aliviar os sintomas próprios da fase.

Verificou-se que os homens pouco sabem a respeito da menopausa, e que o período do climatério é predominantemente desconhecido, o que inviabiliza o apoio à mulher. É importante ressaltar que não se evidenciou que a escolaridade interfere no conhecimento de climatério e menopausa. 
Em face ao exposto, evidencia-se a necessidade de mais estudos que busquem explorar a temática menopausa/ climatério. Nesse sentido, o presente estudo apresenta relevância não apenas para a saúde da mulher, mas também para a saúde coletiva. Além disso, o estudo avança ao incluir a visão masculina, pois esta pode contribuir para que a mulher vivencie as referidas fases com mais segurança, apoio, companheirismo e solidariedade.

\section{REFERÊNCIAS}

1. Instituto Brasileiro de Geografia e Estatística [homepage na internet]. Quantidade de homens e mulheres: Porcentagem da população, por sexo [acesso em $14 \mathrm{dez}$ 2018]. Disponivel em: https://educa.ibge.gov.br/jovens/ conheca-o-brasil/populacao/18320-quantidade-de-homens-emulheres.html.

2. Ministério da Saúde (BR). Secretaria de Atenção à Saúde. Departamento de Ações Programáticas Estratégicas.Política Nacional de Atenção Integral à Saúde da Mulher-Princípios e Diretrizes [Internet]. 2004. [acesso em 19 mar 2019]. Disponivel em : http://bvsms.saude.gov. br/bvs/publicacoes/politica_nac_atencao_mulher.pdf

3. Ministério da Saúde (BR). Secretaria de Atenção à Saúde. Departamento de Ações Programáticas Estratégicas. Manual de atenção à mulher no climatério/menopausa [Internet]. 2008 [acesso em 27 dez 2018]. Disponivel em: http://bvsms.saude.gov.br/bvs/publicacoes/ manual_atencao_mulher_climaterio.pdf

4. Souza NLSA.de, Araújo CL de O. Marco do envelhecimento feminino, a menopausa: sua vivência, em uma revisão de literatura. Revista Kairós Gerontologia [Internet]. 2015 [acesso em 21 nov 2018] 18(2):149-165. Disponivel em: http://ken.pucsp.br/kairos/article/ view/26430/18952

5. Fairclough N. Discurso e mudança social. Brasilia: Editora Universidade de Brasília. 2001.

6. Yin RK. Estudo de Caso: Planejamento e Métodos. Bookman editora, 2015. 290p.

7. Lomônacoa C, Tomaza RAF, Ramosa MT de O. O impacto da menopausa nas relações e nos papéis sociais estabelecidos na família e no trabalho. Reprodução \& Climatério [Internet].2015 [acesso em 21 nov 2018] 30(2):
58-66. Disponivel em: https://www.sciencedirect.com/ science/article/pii/S1413208715000412

8. Moreira MA, Braitt LL. As alterações biopsicossociais no climatério e a inter-relação com a qualidade de vida: um estudo de revisão integrativa. Memorialidades [ Internet]. 2014 [acesso em 19 nov 2018] 11(21): 57-83. Disponivel em: http://periodicos.uesc.br/index.php/ memorialidades/article/view/628

9. Silva MA, Franceschi RJ, Paganotto M, Passoni CRMS. Perfil nutricional e sintomatológico de mulheres no climatério e menopausa. Caderno da Escola de Saúde [Internet]. 2017 [acesso em 15 nov 2018] 8:96113. Disponivel em: http://portaldeperiodicos.unibrasil.com.br/index.php/cadernossaude/article/viewFile/2360/1932

10. Ferreira ICC, Silva SS, Almeida RS de. Menopausa, Sinais e Sintomas e seus Aspectos Psicológicos em Mulheres sem Uso de Reposição Hormonal. Ensaios e Ciência: Ciência Biológicas, Agrárias e da Saúde [Internet]. 2015 [acesso em 10 nov 2018] 19(2). Disponivel em: https://www.redalyc.org/articulo.oa?id=26042168003

11. Nogueira JS, Oliveira BS, Mamede MV, Silva LDC. Sintomas psicológicos em mulheres climatéricas cardiopatas. Cogitare Enfermagem [Internet]. 2018 [acesso em 21 nov 2018] (23)2. Disponivel em: http://www.saude. ufpr.br/portal/revistacogitare/wp-content/uploads/ sites/28/2018/05/54075-233987-1-PB.pdf

12. Leite MT, Taschetto A, Hildebrandt LM, Sand ICPVO. homem também fala: o climatério feminino na ótica masculina. Revista Eletrônica de Enfermagem [Internet]. 2013 [acesso em 21 nov 2018] 15(2):344-51. Disponivel em: https://www.revistas.ufg.br/fen/article/ view/15424/14785 